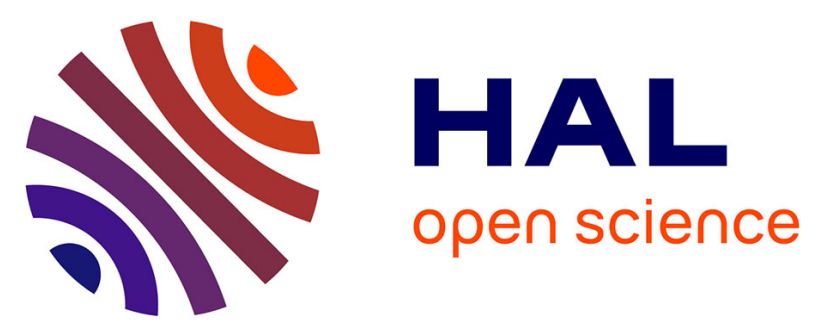

\title{
Intelligent and Accessible Data Flow Architectures for Manufacturing System Optimization
}

Roby Lynn, Aoyu Chen, Stephanie Locks, Chandra Nath, Thomas Kurfess

\section{To cite this version:}

Roby Lynn, Aoyu Chen, Stephanie Locks, Chandra Nath, Thomas Kurfess. Intelligent and Accessible Data Flow Architectures for Manufacturing System Optimization. IFIP International Conference on Advances in Production Management Systems (APMS), Sep 2015, Tokyo, Japan. pp.27-35, 10.1007/978-3-319-22756-6_4. hal-01417394

\section{HAL Id: hal-01417394 \\ https://hal.science/hal-01417394}

Submitted on 15 Dec 2016

HAL is a multi-disciplinary open access archive for the deposit and dissemination of scientific research documents, whether they are published or not. The documents may come from teaching and research institutions in France or abroad, or from public or private research centers.
L'archive ouverte pluridisciplinaire HAL, est destinée au dépôt et à la diffusion de documents scientifiques de niveau recherche, publiés ou non, émanant des établissements d'enseignement et de recherche français ou étrangers, des laboratoires publics ou privés. 


\title{
Intelligent and Accessible Data Flow Architectures for Manufacturing System Optimization
}

\author{
Roby Lynn, Aoyu Chen, Stephanie Locks, Chandra Nath, Thomas Kurfess* \\ roby.lynn@gatech.edu,achen75@gatech.edu,slocks@gatech.edu,nathc2@asme.org, \\ *kurfess@gatech.edu \\ The George W. Woodruff School of Mechanical Engineering, Georgia Institute of Technology, \\ Atlanta, GA 30309, USA
}

\begin{abstract}
Many traditional data acquisition (DAQ) systems are expensive and inadaptable - most rely on traditional closed-source platforms - thus limiting their usefulness for machine tool diagnostics, process control and optimization. In this study, three different intelligent data flow architectures are designed and demonstrated based on consumer grade off-the-shelf hardware and software. These architectures allow data flow between both open- and closed-source platforms through multiple wired and wireless communication protocols. The proposed architectures are also evaluated for machine tool diagnostics and monitoring of multiple machine tools in manufacturing systems. To realize cloud-based manufacturing, real time sensor data are collected and displayed on remote interfaces, smart devices and a cloud/global data platform via the Internet. Findings reveal that such cyber physical system (CPS)-based manufacturing systems can effectively be used for real time process control and optimization.
\end{abstract}

KEYWORDS: Intelligent manufacturing, Machine communications, Data flow, Machine diagnostics, Productivity

\section{INTRODUCTION}

Nowadays, in advanced high-tech manufacturing systems, much attention is given to big data analytics for better process control and optimization. When multiple machines and devices are involved in complex manufacturing systems for a single part to be produced, information has to be transferred between these components to enable efficient system operation. In such a case, CPS-based digital manufacturing can enable better process control by realizing an open accessible data flow and exchange between devices $[1,2]$. Although high performance DAQ systems are available that can enable these process improvements, many of these systems are found to be both inaccessible and inadaptable due to their cost and reliance on closed-source platforms. In addition, the sensors used in most of these systems are hard wired, resulting in inflexibility.

Within digital manufacturing, a few data flow architectures have been proposed to realize data acquisition and cloud storage [3-5]. Some research were conducted to address the data accessibility limitations of the standard machine diagnostics approach by integrating a data flow architecture [6]. Shen et al. presented a concept of iShopFloor- 
an intelligent shop floor based on the Internet, web, and agent technologies supported by Java and XML [7]. The generic architecture makes the system respond quickly to shop floor changes and customer demands. However, the Java based communication between agents limits the hardware platform. Also, the Java applets' user interface decreases the flexibility of data manipulation. Wang also proposed a cloud-based approach to distributed process planning based on machine and resource availability [8]. $\mathrm{Xu}$ et al. designed an economic wireless sensor network system for structural health monitoring using off-the-shelf hardware [9]. This approach is novel, but is not able to transmit raw sensor data in real-time and the user must manually collect it from the system for later analysis.

Thanks to the maker movement, the limitations of the previously performed works in industrial data flow can be addressed by employing readily available and affordable high performance consumer-grade microcontrollers, sensors and wireless communication modules. While the computing power available on these platforms is limited, it can be harnessed to perform data analysis on the device itself before the data are relayed on to a cloud-based storage system. Many of these hardware platforms are open-source, and can thus be easily modified to adapt in evolving production environments. The simple connectivity of this hardware can enable the development of a novel DAQ system that is interconnected and able to share data across platforms and facilities. Additionally, the accessibility of this hardware could allow for increased adoption by manufacturers for whom traditional DAQ systems were either unusable due to their inadaptability or inaccessible due to their cost. However, the construction of an intelligent data flow architecture using these widely available components has yet to be formulated in modern manufacturing systems.

The objective of this research is to design and evaluate intelligent, accessible, and low-cost industrial data flow architectures for manufacturing system control and optimization. In this paper, three different architectures are designed using high performance consumer-grade hardware and software systems that allow data flow between both open- and closed-source platforms. A cloud-based manufacturing system is demonstrated using multiple wired and wireless communication protocols. These architectures are also evaluated for machine tool diagnostics and monitoring of multiple machine tools in manufacturing systems.

\section{DATA FLOW SYSTEM ARCHITECTURES}

Collaborative manufacturing and real-time data collection and analysis are of great interest in the emerging CPS field. The overall purpose of the data flow system architecture presented below is to enable collaborative analysis and optimization by making manufacturing process data from different machine tools widely available. Three different data flow system methodologies are constructed and evaluated: 1) MTConnect network, 2) wireless distributed sensor network, and 3) sensor-MTConnect hybrid network. This collection of wireless protocols, various processor architectures, and sensor types demonstrates that the architecture is portable between all platforms. In this section, all system architectures are discussed with construction methodology, capabilities, limitations, and a case study in the machining environment. 


\subsection{MTCONNECT NETWORK}

SYSTEM ARCHITECTURE. A connectivity concept to a piece of manufacturing equipment is created using MTConnect and a National Instruments (NI) myRIO fieldprogrammable gate array (FPGA) device. MTConnect - a communication protocol for data acquisition from CNC machine tools - is implemented as a web service, enabling any web browser to access the machine tool's data output via a wired or Wi-Fi router. The myRIO is connected to the local wireless network and used to generate HTTP requests that are sent to the machine tool. The MTConnect-based data flow architecture system is presented in Fig. 1. The myRIO processes the MTConncet data from the machine tool and presents them on a display.

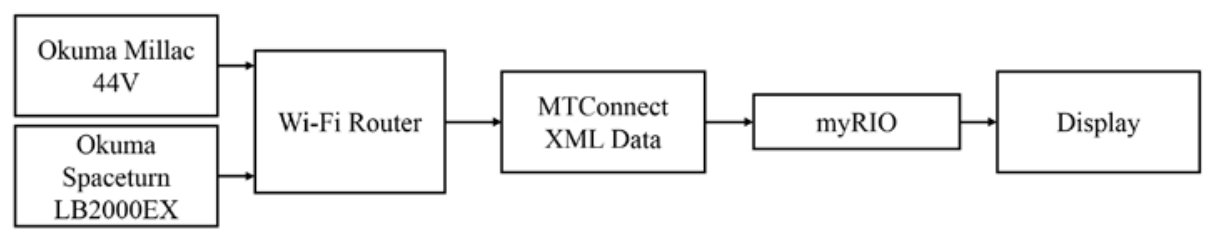

Fig. 1. MTConnect Data Flow

CASE STUDY: PROCESS CONDITION MONITORING. The MTConnect implementation allows for access to spindle speed, spindle motor power output, axis positions and axis servo power outputs of machine tool(s). These data are gathered from two machine tools simultaneously: an Okuma Millac M44V and an Okuma Spaceturn LB2000EX.

LOCAL INTERFACE. The axis loads and positions from the machine tools are graphed and displayed to the user on a computer display as shown in Fig. 2. The visualization of these data allows manufacturers to quickly and easily monitor and obtain an intuitive idea of the process state, the tool-work relative position, and the machining process parameters.

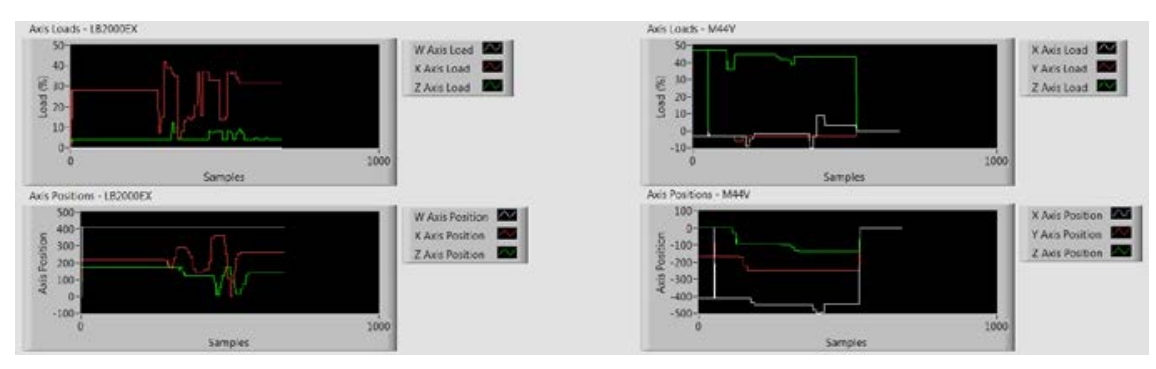

Fig. 2. MTConnect Front Panel

MTCONNECT CAPABILITIES AND LIMITATIONS. The MTConnect protocol provides manufacturers with a simple yet powerful way to access data collected by their 
machine tools. Because the protocol is implemented as a web service, it is easily accessible to any device that is connected to the machine network. Due to the protocol's reliance on the machine controller for the data, both the update speed and the number of available data are limited. For example, most CNC machine tools do not include accelerometers in their spindles to monitor vibration, and thus these data are not available from MTConnect. Additionally, the data update speed is set by the controller and therefore it may not be fast enough for some applications. For the Okuma machine tools utilized in this implementation, the maximum data update rate is $1 \mathrm{~Hz}$; this may be too slow to enable real-time data analysis using MTConnect data alone.

\subsection{DISTRIBUTED WIRELESS SENSOR NETWORK}

SYSTEM ARCHITECTURE. Two related configurations are created to demonstrate the distributed wireless sensor architecture: one relies on ZigBee and Wi-Fi, while the other relies on Bluetooth Low Energy (BLE) and Wi-Fi. The configurations are inspired by the internet-of-things (IoT) philosophy that is nowadays gaining widespread adoption in both industrial and consumer applications. To increase the accessibility of the system, consumer grade microcontrollers and sensors are employed. Both demonstrations of the architecture use an accelerometer placed on a CNC machine tool to monitor vibration.

The first construction of this architecture uses a PIC32MX board, a Microchip WiFi module and Digi's 802.14 XBee radios. The PIC32MX is used to gather accelerometer data via the ZigBee link and transmit them via Wi-Fi to a receiving device. This implementation highlighted the use of multiple wireless networking standards that can be chained together to create a coherent data pathway. The connection diagram of the PIC32 implementation is presented in Fig. 3. The PIC32 serves to receive the accelerometer data and package it so that it can be interpreted by the server PC after flowing through the Wi-Fi connection; the PC then renders the data to users through the HTTP server. The NFC tag is used by networked smart devices to access the HTTP server: the IP address of the server is written to the tag, and devices access the page through a mobile browser after reading from the tag.

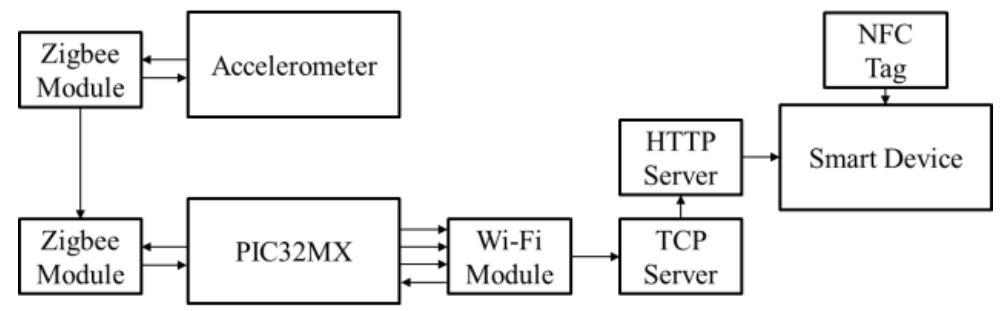

Fig. 3. Data Flow in PIC32 IoT Configuration

This architecture is then reconfigured using different communication methods and illustrates the use of a different device, the NI myRIO. By replacing the PIC32 with a myRIO device and swapping the ZigBee chips for BLE chips, a new system with the 
same capabilities as the ZigBee and PIC32 system is established. Once again, the system uses only consumer grade off-the-shelf components and illustrates the ease of connectivity offered by current technologies.

CASE STUDY: MACHINE CRASH DETECTION. The wireless sensor network is employed to monitor the vibration generated by the spindle that arises from a crash of a CNC machine tool. By monitoring the accelerometer output, normal spindle operation can be distinguished from a machine crash or other emergency condition.

LOCAL INTERFACE. Due to the increasing prevalence of Wi-Fi networks, 802.11g was chosen as the backbone network type for data flow for the wireless sensor system. To increase the accessibility of the data from a variety of connected devices, a dynamic webpage was created that was compatible with both standard and mobile browsers.

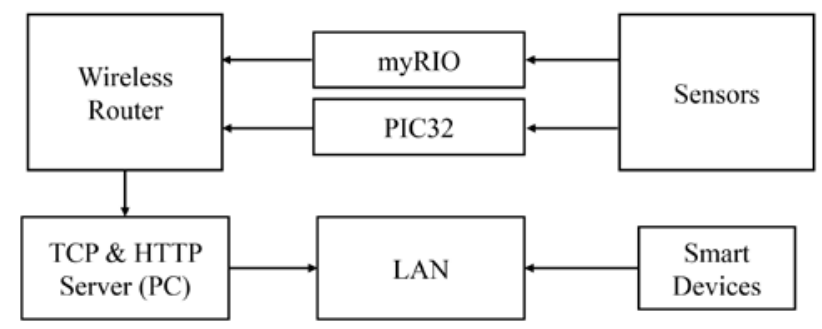

Fig. 4. Dynamic Webpage Data Flow

The webpage was created using HTML5, JavaScript and the JavaScript Data-Driven Documents (D3) library. The D3 library is responsible for rendering the graph of sensor data coming from the networked microcontrollers. The overview of this data flow architecture is shown in Fig. 4. Because the webpage is hosted on the local network, it is visible to other connected smart devices. This interface is easily accessed by smart devices by exploiting the quick tap-and-go abilities of an RFID communication link. When a user wants to view process data from a particular machine tool, they only need to tap an NFC-capable device on the tag to access the dynamic webpage.

CLOUD-BASED REMOTE DATA INTERFACE. To demonstrate the wide availability of these data, they are streamed to an active cloud data visualization tool via the Internet where they are accessible from any device. This interface utilized Google Docs' spreadsheet environment to visualize incoming sensor data. A TCP server was written in C\# that received the sensor output stream from the PIC32 microcontroller and parsed it into individual readings. The Google Data API for .NET was then invoked within the C\# server program to communicate with a Google spreadsheet. While not as responsive as the local webpage, the Google Doc implementation proved that these data can be accessible from anywhere using an internet-enabled device.

CAPABILITIES AND LIMITATIONS. The wireless sensor network described above is useful in production environments where data availability and quick implementation are critical; however, because the system relies heavily on the relatively 
weak computing power of standard microcontrollers, the data throughput is limited. For this reason, the wireless sensor network may not be suitable for applications in which high-frequency data analysis must be performed in real-time.

\subsection{SENSOR-MTCONNECT HYBRID NETWORK}

SYSTEM ARCHITECTURE. The spindle is a key component for a CNC and its sudden break down usually causes a huge loss of productivity. Using a real-time data acquisition system, diagnostics can be performed on a CNC machine tool to evaluate the health of the spindle to avoid this productivity loss. This implementation demonstrates the usefulness of a distributed sensor approach combined with the MTConnect protocol. Machine diagnostics based on the combination of external sensors and MTConnect have not been fully studied yet, so this implementation attempts to demonstrate the novelty of the approach.

The data flow architecture for spindle diagnostics is shown in Fig. 5. An accelerometer, a power meter, and MTConnect data are combined together and processed by an NI myRIO. Both the accelerometer and the power meter are hard wired to the processor to demonstrate the concept of the data flow architecture and the effectiveness of the diagnostic method. The diagnostics result appears as an FFT pattern. Using JavaScript, the data can be transferred in real-time to cloud-based dynamic documents such as Google Docs to enable further analysis on remote systems.

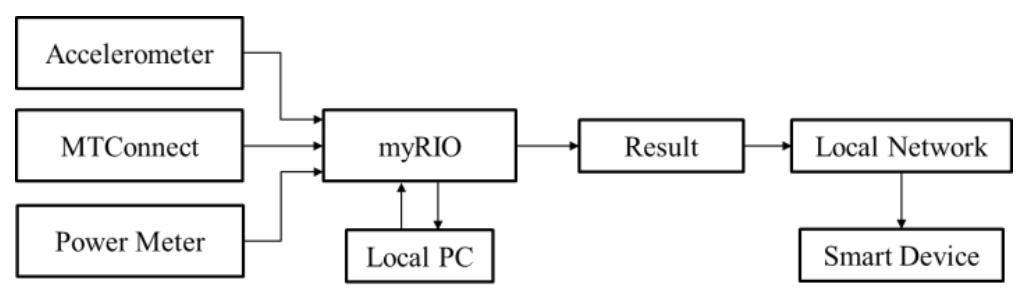

Fig. 5. Spindle Diagnostics Data Flow

CASE STUDY: MACHINE TOOL SPINDLE DIAGNOSTICS. Accelerometers are attached to the spindle housing of an Okuma Millac M44V to assess and diagnose the health of the spindle. From the vibration data of the accelerometer, a fast Fourier transform (FFT) is performed to provide information related to critical elements of the spindle, including the spindle bearings, which are typical components that fail due to lifecycle fatigue or a severe crash scenario [10]. Accelerometer signals have certain well defined characteristics, such as their fundamental frequency, as well as certain harmonics that are integer multiples of the fundamental frequency. Other well documented characteristics include bearing related frequencies or so-called bearing tones, such as the ball pass frequency on the rollers and the races [11]. A record of a healthy spindle FFT is calculated and stored for the spindle at various spindle operational frequencies for comparison purposes. Spindle health degradation is monitored by comparing the record of the initial healthy spindle to the vibration of the current spindle. 
LOCAL INTERFACE. It should be noted that the current system does not have a permanent human interface device. By allowing the workers to use their pre-existing smart devices or by providing them with tablets, the cost of having a full computer and display at each machine can be eliminated. The local interface can be transmitted to the cloud if desired, making the operation of this system truly global. If standard communication protocols such as MTConnect, Bluetooth and Wi-Fi are employed, updating the systems to employ the latest interface technology will be as easy as updating one's smartphone. Finally, while the communication for this system is one way, in the future, two way communication may be implemented that integrates the machine controller into automatic test cycles based on a pre-set time or system conditions.

SPINDLE DIAGNOSTICS CAPABILITIES AND LIMITATIONS. The spindle diagnostics example illustrates the flexibility and ease with which data from manufacturing systems can be processed and monitored by the data flow architecture. The fusion of MTConnect and discrete sensors helps to increase the diversity of available data. Based on time and frequency domain analysis, a malfunction of the spindle can be detected. Messages can be transmitted to alert operators and maintenance and scheduling personnel to take further actions. Data can be shared on smart devices or to remote PCs through local networks or the cloud via the Internet.

\section{DISCUSSION}

This intelligent data flow architecture demonstrates that multiple configurations exist which can be employed to create a data pathway between sensors and data users. In the distributed wireless sensing approach, low-cost sensors and computing platforms can be deployed to monitor machine tools on the shop floor. This system is accessible to any machine tool operator because it is both affordable and easy to implement. When combined with Google Docs, the wireless sensor data can be shared between facilities and used for collaborative manufacturing. This system is unique in that it relies on accessible and easily deployable consumer grade hardware; thus, widespread adoption in smaller facilities is more likely.

While dedicated computer displays were employed in the preceding implementations to present both raw and analyzed data to users, they are not required in order to realize these data flow architectures. As smart devices such as phones and tablets become more affordable and widespread, it is reasonable to expect that all personnel working in a given manufacturing facility would carry such a device. As such, the dedicated displays can be eliminated and each smart device can serve as a separate display for its owner. These personal displays would allow for a limitless number of users to view one set of data at a time; if the data were stored in the cloud, these data could be accessed from anywhere. Cyber-security concerns present problems that must be solved in order to advance this concept, but this has been proven to be possible by several successful cloud-based storage services that exist today.

The implementation of a reconfigurable data flow architecture, such as the ones presented above, would be highly adaptable to changes on the shop floor and would require only simple software updates (which can be performed remotely) to alter system behavior. A reconfigurable system would allow manufacturers access to any process or 
machine data that they want to analyze, which eliminates costly and time-consuming hardware setups. As the speed of data acquisition and analysis can increase with these reconfigurable systems, and if cloud-based storage and processing are integrated, realtime collaborative manufacturing and control begin to look like a reality. This globalization of these manufacturing data would accelerate the pace of process development and optimization, leading to increased productivity, higher sustainability, and lower costs for all participating parties.

\section{CONCLUSIONS}

In this study, three different intelligent and accessible data flow architectures are designed and implemented for manufacturing systems. Conclusions are as follows: i) The MTConnect protocol was shown to be compatible with the myRIO and useful data were successfully gathered and presented, ii) A distributed wireless sensor approach was shown using two different computing platforms - PIC32 and myRIO, iii) A cloud-based data platform was implemented with the wireless sensor approach to demonstrate that the collected data are available globally, and iv) A real-time spindle diagnostics application was developed using the myRIO to demonstrate sophisticated analysis that can be performed with the data acquired using one of these architectures.

\section{REFERENCES}

1. Rajkumar, R., et al. Cyber-Physical Systems: The Next Computing Revolution. in Design Automation Conference (DAC), 2010 47th ACM/IEEE. 2010.

2. Wang, L., et al., Current Status and Advancement of Cyber-Physical Systems in Manufacturing. Journal of Manufacturing Systems, 2015. 36.

3. Karnouskos, S., et al., A SOA-based Architecture for Empowering Future Collaborative Cloud-based Industrial Automation. IECON 2012 - 38th Annual Conference on IEEE Industrial Electronics Society, 2012.

4. Tao, F., et al., Cloud Manufacturing: A Computing and Service-Oriented Manufacturing Model. Journal of Engineering Manufacture, 2011. 225(10): p. 8.

5. Wang, X.V. and X.W. Xu, ICMS: A Cloud-Based Manufacturing System. Cloud Manufacturing, 2013.

6. Milfelner, M., et al., An Overview of Data Acquisition System for Cutting Force Measuring and Optimization in Milling. Journal of Materials Processing Technology, 2005. 164-165: p. 8.

7. Shen, W., et al., iShopFloor: an Internet-Enabled Agent-Based Intelligent Shop Floor. IEEE Transactions on Systems, Man, and Cybernetics, 2005. 35(3): p. 11.

8. Wang, L., Machine Availability Monitoring and Machining Process Planning towards Cloud Manufacturing. CIRP Journal of Manufacturing Science and Technology, 2013. 6(4): p. 11.

9. Xu, N., et al., A Wireless Sensor Network for Structural Monitoring. Proceedings of the 2nd International Conference on Embedded Networked Sensor Systems, 2004: p. 12.

10. Li, Y., et al., Adaptive Prognostics for Rolling Element Bearing Condition. Mechanical Systems and Signal Processing, 1999. 13(1).

11. Billington, S., Sensor and Machine Condition Effects in Roller Bearing Diagnostics. 1997. 